\title{
Innovative Structured Matrices
}

\author{
Rahul Gupta, Garimella Rama Murthy \\ IIIT-Hyderabad,Gachibowli, Hyderabad, India \\ Email: rahulg583@gmail.com, rammurthy@iiit.ac.in
}

Received February 17, 2013; revised March 17, 2013; accepted April 2, 2013

Copyright (C) 2013 Rahul Gupta, Garimella Rama Murthy. This is an open access article distributed under the Creative Commons Attribution License, which permits unrestricted use, distribution, and reproduction in any medium, provided the original work is properly cited.

\begin{abstract}
Various directions of obtaining novel structured matrices are discussed. A new class of matrices, called "the L-family" matrices are introduced and their properties are studied.
\end{abstract}

Keywords: Innovative Matrices; L Matrices; Structured Matrices

\section{Introduction}

Linear algebra is central to modern mathematics and has been found many applications in Science, Technology, Engineering and many other disciplines. Matrices with special kind of structure like Toeplitz, Hankel etc., are studied with great interest [2]. In this paper, a special family (called "The L-family") of matrices are discussed in detail with some interesting properties. It is expected that this family of matrices will find interesting applications in various disciplines of human endeavour.

This idea of analysing new structured matrices was adopted from Dr. G. Rama Murthy's journal paper "Innovative Structured Matrices", International Journal of Algorithms, Computing and Mathematics Volume 2, Number 4, November 2009.

\section{Logical Idea behind Structured Matrices}

We can think of innovative structured matrices in many ways. For example, one way is to construct a matrix from the indices or subscripts of elements of the matrix. The other way is to assign a particular same value to all elements for each subset of the matrix, where these subsets are taken to be mutually exclusive and exhaustive $[1,3]$.

Constructing matrices from indices point-of-view:

$$
\left(\begin{array}{lll}
a_{11} & a_{12} & a_{13} \\
a_{21} & a_{22} & a_{23} \\
a_{31} & a_{32} & a_{33}
\end{array}\right)
$$

we can map $a_{x y}$ to a function of $x, y, f(x, y)$.

$x, y=1,2,3$ i.e., $a_{x y}=f(x, y)$

The following is the matrix constructed by taking

$$
f(x, y)=x^{2} y+x y^{2}
$$

$$
\left(\begin{array}{ccc}
2 & 6 & 12 \\
6 & 16 & 30 \\
12 & 30 & 54
\end{array}\right)
$$

We can also take $a_{x y}=a f(x, y)$ just like $f(x, y)=|x-y|$ as in a Toeplitz.

Constructing matrices from subset point-of-view:

Let us look at some typical examples.

Example: 1

$$
\left(\begin{array}{lllll}
a_{3} & a_{3} & a_{3} & a_{3} & a_{3} \\
a_{3} & a_{2} & a_{2} & a_{2} & a_{3} \\
a_{3} & a_{2} & a_{1} & a_{2} & a_{3} \\
a_{3} & a_{2} & a_{2} & a_{2} & a_{3} \\
a_{3} & a_{3} & a_{3} & a_{3} & a_{3}
\end{array}\right)
$$

$\Rightarrow$ constructed by taking size-increasing, mutually exclusive and exhaustive square shaped subsets

Example: 2

$$
\begin{array}{ll}
\left(\begin{array}{llll}
a_{4} & a_{3} & a_{2} & a_{1} \\
a_{4} & a_{3} & a_{2} & a_{2} \\
a_{4} & a_{3} & a_{3} & a_{3} \\
a_{4} & a_{4} & a_{4} & a_{4}
\end{array}\right) & \left(\begin{array}{llll}
a_{1} & a_{2} & a_{3} & a_{4} \\
a_{2} & a_{2} & a_{3} & a_{4} \\
a_{3} & a_{3} & a_{3} & a_{4} \\
a_{4} & a_{4} & a_{4} & a_{4}
\end{array}\right) \\
\left(\begin{array}{llll}
a_{4} & a_{4} & a_{4} & a_{4} \\
a_{4} & a_{3} & a_{3} & a_{3} \\
a_{4} & a_{3} & a_{2} & a_{2} \\
a_{4} & a_{3} & a_{2} & a_{1}
\end{array}\right) & \left(\begin{array}{llll}
a_{4} & a_{4} & a_{4} & a_{4} \\
a_{3} & a_{3} & a_{3} & a_{4} \\
a_{2} & a_{2} & a_{3} & a_{4} \\
a_{1} & a_{2} & a_{3} & a_{4}
\end{array}\right)
\end{array}
$$

The above 4 matrices are constructed by taking $L$,$\lrcorner ,$ 
\urcorner , $\Gamma$ shaped subsets of the matrix as the criteria respectively.

Example: 3

$$
\begin{array}{ll}
\left(\begin{array}{llll}
a_{2} & a_{2} & a_{2} & a_{2} \\
a_{2} & a_{1} & a_{1} & a_{2} \\
a_{2} & a_{1} & a_{1} & a_{2} \\
a_{2} & a_{1} & a_{1} & a_{2}
\end{array}\right) & \left(\begin{array}{llll}
a_{2} & a_{2} & a_{2} & a_{2} \\
a_{2} & a_{1} & a_{1} & a_{1} \\
a_{2} & a_{1} & a_{1} & a_{1} \\
a_{2} & a_{2} & a_{2} & a_{2}
\end{array}\right) \\
\left(\begin{array}{llll}
a_{2} & a_{2} & a_{2} & a_{2} \\
a_{1} & a_{1} & a_{1} & a_{2} \\
a_{1} & a_{1} & a_{1} & a_{2} \\
a_{2} & a_{2} & a_{2} & a_{2}
\end{array}\right) & \left(\begin{array}{llll}
a_{2} & a_{1} & a_{1} & a_{2} \\
a_{2} & a_{1} & a_{1} & a_{2} \\
a_{2} & a_{1} & a_{1} & a_{2} \\
a_{2} & a_{2} & a_{2} & a_{2}
\end{array}\right)
\end{array}
$$

These matrices are constructed by taking $\downarrow$ shaped subsets of the matrix as the criteria in all the four directions with opening towards south, east, west and north directions respectively.

Remark: This logical approach can be extended to arrive at large number of structured matrices (like Toeplitz matrix) [3].

The L-family of matrices:

Now we focus our attention on the class of matrices in Example 2.

This class of matrices can be called "The L-family" matrices due to their resemblance in their structure with the letter "L".

$$
\left(\begin{array}{llll}
a_{4} & a_{3} & a_{2} & a_{1} \\
a_{4} & a_{3} & a_{2} & a_{2} \\
a_{4} & a_{3} & a_{3} & a_{3} \\
a_{4} & a_{4} & a_{4} & a_{4}
\end{array}\right)
$$

L-matrix

$$
\left(\begin{array}{cccc}
a_{4} & a_{4} & a_{4} & a_{4} \\
a_{4} & a_{3} & a_{3} & a_{3} \\
a_{4} & a_{3} & a_{2} & a_{2} \\
a_{4} & a_{3} & a_{2} & a_{1}
\end{array}\right)
$$

inv-L matrix

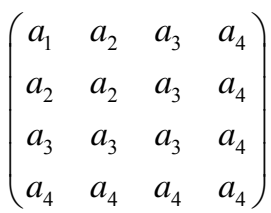

rev-L matrix

$$
\left(\begin{array}{llll}
a_{4} & a_{4} & a_{4} & a_{4} \\
a_{3} & a_{3} & a_{3} & a_{4} \\
a_{2} & a_{2} & a_{3} & a_{4} \\
a_{1} & a_{2} & a_{3} & a_{4}
\end{array}\right)
$$

rev-inv-L matrix [rev: stands for reverse and inv: stands for inverse].

Let us consider only square matrices in our entire discussion.

Let us define an originator of a matrix in L-family. The $i^{\text {th }}$ originator of a $n^{*} n$ L-family matrix is the element which occurs (2i-1) times in the matrix.

In all the above mentioned matrices, $a_{1}-1^{\text {st }}$ originator, $a_{2}-2^{\text {nd }}$ originator, $a_{3}-3^{\text {rd }}$ originator and $a_{4}-4^{\text {th }}$ originator.

Let us examine some of the properties of L-family:

Claim 1: For any L-family matrix $A,\|A\|_{1}=\|A\|_{\infty}$ where $\|$.$\| represents natural norm [4].$

\section{Proof 1:}

$$
\|A\|_{1}=\max _{\|z\|_{1}=1}\left\|A_{z}\right\|_{1}=\max _{1 \leq j \leq n}\left\{\sum_{i=1}^{m}\left|a_{i j}\right|\right\}
$$

$=$ maximum absolute column sum of the matrix

$$
\|A\|_{\infty}=\max _{\|z\|_{\infty}=1}\left\|A_{z}\right\|_{\infty}=\max _{1 \leq j \leq m}\left\{\sum_{i=1}^{n}\left|a_{i j}\right|\right\}
$$

$=$ maximum absolute row sum of the matrix

For rev-L and inv-L matrices absolute kth row sum is equal to absolute kth column sum for $k=1,2, \cdots, n$. For $\mathrm{L}$ - and rev-inv-L matrices absolute kth column sum is equal to absolute $(n-k+1)^{\text {th }}$ column sum for $k=1,2, \cdots, n$. (Note: $m=n$ for a square matrix).

Hence $\|A\|_{1}=\|A\|_{\infty}$

Claim 2: For an L-family matrix to be stochastic, all the originators of it must be equal to each other.

Proof 2: The sum of all the elements in each column of a stochastic matrix is equal to 1.Consider an L-matrix of order " $n$ ", i.e., $\left\{a_{i}\right\}, i=1,2, \cdots, n$ are originators.

Sum of all the elements in $1^{\text {st }}$ column $=n a_{n}$

$$
n a_{n}=1 \Rightarrow a_{n}=1 / n
$$

Sum of all the elements in $2^{\text {nd }}$ column $=$ $(n-1) a_{n-1}+a_{n}$

$$
\Rightarrow(n-1) a_{n-1}+1 / n=1 \Rightarrow a_{n-1}=1 / n
$$

Sum of all the elements in $3^{\text {rd }}$ column $=$

$$
\begin{aligned}
(n-2) a_{n-2} & +a_{n-1}+a_{n} \\
& \Rightarrow(n-2) a_{n-2}+2 / n=1 \Rightarrow a_{n-2}=1 / n
\end{aligned}
$$

Similarly, sum of all the elements in $k^{\text {th }}$ column $=$ $(n-k+1) a_{n-k+1}+(k-1) / n$

$$
\begin{aligned}
& \Rightarrow(n-k+1) a_{n-k+1}+(k-1) / n=1 \\
& \Rightarrow a_{n-k+1}=1 / n \quad \text { for } k=4,5, \cdots n
\end{aligned}
$$

Therefore, $a_{i}=1 / n$ for all $i=1,2, \cdots, n$

Hence all the originators must be equal to each other.

Similar type proof can be provided for other type of matrices rev-L, inv-L, rev-inv-L matrices also.

Claim 3: The determinant of a rev-L or inv-L matrix with originators $\left\{a_{i}\right\}, i=1,2, \cdots, n$ is equal to $a_{n}\left(a_{1}-a_{2}\right)\left(a_{2}-a_{3}\right)\left(a_{3}-a_{4}\right) \cdots\left(a_{n-1}-a_{n}\right)$.

Proof 3: The proof for rev-L matrix is as follows.

Perform the following elementary row operations on the determinant.

1) $R_{i} \rightarrow R_{i}-R_{i+1}$ for all $i=1,2, \cdots,(n-1)$

2) Take $a_{n},\left(a_{1}-a_{2}\right),\left(a_{2}-a_{3}\right), \cdots,\left(a_{n-1}-a_{n}\right)$ common out of the determinant

3) $R_{i} \rightarrow R_{i}-R_{i-1}$ for all $i=n,(n-1), \cdots, 2$

4) The remaining determinant goes to " 1 " as it is Identity matrix. 
Hence proved for rev-L matrix.

The proof for inv-L matrix is as follows.

1) $R_{i} \rightarrow R_{i}-R_{i-1}$ for all $i=n,(n-1), \cdots, 2$

2) Take $a_{n},\left(a_{1}-a_{2}\right),\left(a_{2}-a_{3}\right), \ldots\left(a_{n-1}-a_{n}\right)$ common out of the determinant

3) $R_{i} \rightarrow R_{i}-R_{i+1}$ for all $i=1,2, \cdots,(n-1)$

4) The remaining determinant goes to " 1 " as it is Identity matrix.

Hence proved for inv-L matrix also.

Claim 4: The determinant of L-matrix or rev-inv-L matrix with originators $\left\{a_{i}\right\}, i=1,2, \cdots, n$ is equal to

$$
(-1)^{[n / 2]} a_{n}\left(a_{1}-a_{2}\right)\left(a_{2}-a_{3}\right)\left(a_{3}-a_{4}\right) \cdots\left(a_{n-1}-a_{n}\right),
$$

where [.] denotes step function/greatest integer function.

The above claim can be easily proved using a simple mathematical induction. Before going through the proof let us look at some criteria which will be useful in proving the claim.

Let us define Mirror image of a $n^{*} n$ ordered square matrix $A=\left[a_{(i)(j)}\right]$ as the matrix $B=\left[b_{(i)(j)}\right]$, where $b_{(i)(j)}=a_{(i)(n+1-j)}$.

$$
\left(\left.\begin{array}{lll}
a_{11} & a_{12} & a_{13} \\
a_{21} & a_{22} & a_{23} \\
a_{31} & a_{32} & a_{33}
\end{array}\right|_{\text {(MIRROR) }}\left(\begin{array}{lll}
a_{13} & a_{12} & a_{11} \\
a_{23} & a_{22} & a_{21} \\
a_{33} & a_{32} & a_{31}
\end{array}\right)\right.
$$

$$
\left(\begin{array}{lll}
0 & 0 & 1 \\
0 & 1 & 0 \\
1 & 0 & 0
\end{array}\right)\left(\text { say } M_{3}\right) \text { is the mirror image of Identity }
$$

matrix, $\mathrm{I}_{3}$.

Our aim is to find out the determinant (say $D_{n}$ ) of $M_{n}$.

Claim 5: $D_{n}=(-1)^{[n / 2]}$

Proof 5: We shall prove this using mathematical induction method.

Let $D_{k-1}=(-1)^{[(k-1) / 2]}$.

Then

$D_{k}=(-1)^{k+1} D_{k-1}=(-1)^{k+1}(-1)^{[(k-1) / 2]}=(-1)^{k+1+[(k-1) / 2]}$

Case 1: If $k$ is even $(=2 p)$

$$
\begin{aligned}
D_{k} & =(-1)^{2 p+1+[p-1 / 2]}=(-1)^{2 p+1+p-1}=(-1)^{3 p} \\
& =(-1)^{p}=(-1)^{k / 2}=(-1)^{[k / 2]}
\end{aligned}
$$

Case 2: If $k$ is odd $(=2 p+1)$

$$
D_{k}=(-1)^{2 p+1+1+[p]}=(-1)^{p}=(-1)^{[p+1 / 2]}=(-1)^{[k / 2]} \text {. }
$$

Hence, $D_{n}=(-1)^{[n / 2]}$.

The proof for claim 4 is as follows.
Proof 4: The proof for L-matrix is as follows:

Perform the following elementary row operations on the determinant.

5) $R_{i} \rightarrow R_{i}-R_{i+1}$ for all $i=1,2, \cdots,(n-1)$

6) Take $a_{n},\left(a_{1}-a_{2}\right),\left(a_{2}-a_{3}\right), \cdots,\left(a_{n-1}-a_{n}\right)$ common out of the determinant

7) $R_{i} \rightarrow R_{i}-R_{i-1}$ for all $i=n,(n-1), \cdots, 2$

8) The remaining determinant goes to " $D_{n}$ " which is equal to $(-1)^{[\mathrm{n} / 2]}$.

Hence proved for L-matrix.

The proof for rev-inv-L matrix is as follows:

5) $R_{i} \rightarrow R_{i}-R_{i-1}$ for all $i=n,(n-1), \cdots, 2$

6) Take $a_{n},\left(a_{1}-a_{2}\right),\left(a_{2}-a_{3}\right), \ldots\left(a_{n-1}-a_{n}\right)$ common out of the determinant

7) $R_{i} \rightarrow R_{i}-R_{i+1}$ for all $i=1,2, \cdots,(n-1)$

8) The remaining determinant goes to " $D_{n}$ " which is equal to $(-1)^{[n / 2]}$

Hence proved for rev-inv-L matrix also.

Therefore, from the above we can say that any L-family matrix of order $n * n$ will be a non-singular matrix if and only if $\mathrm{n}^{\text {th }}$ originator is non-zero and any $i^{\text {th }}$ generator is not equal to to $(i+1)^{\text {th }}$ originator (for all $i=1,2, \cdots,(n-1))$ matrix.

Claim 5: If we permute $\{a(i)\}$ with its adjacent number i.e. with

$\left\{a_{i-1}\right\}$ or $\left\{a_{i+1}\right\}$ (in circular way), the value of

$$
D_{n}=\Delta(L) \text { changes to } D^{\prime}=\frac{\left\{a_{n-1} \cdot\left(a_{n}-a_{1}\right)\right\}}{\left\{a_{n} \cdot\left(a_{n-1}-a_{n}\right)\right\}} \cdot \Delta(L)
$$

and $D^{\prime \prime}=\frac{\left\{a_{1} \cdot\left(a_{n}-a_{1}\right)\right\}}{\left\{a_{n} \cdot\left(a_{1}-a_{2}\right)\right\}} \cdot \Delta(L)$

\section{Proof 5:}

Case 1. When replacing $a_{i}$ by $a_{i-1}$ for $i=2,3, \cdots, n$ and $a_{1}$ by $a_{n}$ (in Circular Manner)

i.e. $a_{n} \rightarrow a_{n-1}, a_{n-1} \rightarrow a_{n-2}, \cdots, a_{2} \rightarrow a_{1}$ and $a_{1} \rightarrow a_{n}$ then the value of Det. become $D^{\prime}=\frac{\left\{a_{n-1} \cdot\left(a_{n}-a_{1}\right)\right\}}{\left\{a_{n} \cdot\left(a_{n-1}-a_{n}\right)\right\}} \cdot \Delta(L)$ and by dividing it by the actual value of $\Delta(L)$,

$$
\frac{D^{\prime}}{D}=\frac{\left\{a_{n-1} \cdot\left(a_{n}-a_{1}\right)\right\}}{\left\{a_{n} \cdot\left(a_{n-1}-a_{n}\right)\right\}}
$$

Case 2. When replacing $a_{i}$ by $a_{i+1}$ for $i=1,2, \cdots, n-1$ and $a_{n}$ by $a_{1}$ (in Circular Manner)

i.e. $\quad a_{1} \rightarrow a_{2}, \quad a_{2} \rightarrow a_{3}, \cdots, a_{n-1} \rightarrow a_{n}$ and $a_{n} \rightarrow a_{1}$ then the value of Det. become

$D^{\prime \prime}=\frac{\left\{a_{1} \cdot\left(a_{n}-a_{1}\right)\right\}}{\left\{a_{n} \cdot\left(a_{1}-a_{2}\right)\right\}} \cdot \Delta(L)$ and 
by dividing it by the actual value of Det $(L)$,

$$
\frac{D^{\prime \prime}}{D}=\frac{\left\{a_{1} \cdot\left(a_{n}-a_{1}\right)\right\}}{\left\{a_{n} \cdot\left(a_{1}-a_{2}\right)\right\}}
$$

Now note that, if we divide both the ratios,

$$
\frac{D^{\prime}}{D^{\prime \prime}}=\frac{\left\{a_{n-1} \cdot\left(a_{1}-a_{2}\right)\right\}}{\left\{a_{1} \cdot\left(a_{n-1}-a_{n}\right)\right\}}
$$

\section{Block "L" Matrix}

If we take any one of the four kind of $\mathrm{L}$ matrix and make a bigger matrix (having order greater than the previous matrix) which contain the previous matrix then this type of matrix can be characterized as Block "L" where the Matrix has the same building block all over the matrix.

Let us consider a "L" matrix having minimum order (2 $\times 2)-$

$$
\left(\begin{array}{ll}
a & b \\
a & a
\end{array}\right)
$$

Now, we are considering a shape where $b=0$ then $L=\left(\begin{array}{ll}a & 0 \\ a & a\end{array}\right)$ which has a shape of " $L$ "

If we take this matrix and make a new matrix which has this matrix as a building block then

$$
X=\left(\begin{array}{ll}
L & 0 \\
L & L
\end{array}\right)
$$

here $\mathrm{L}$ is the same as described above.

Here we can see that the value of $\Delta(L)=a^{2}$

Again, if we can take $X$ as a building block and if we follow the same shape " $L$ ", we can get a new matrix $L=\left(\begin{array}{cc}X & 0 \\ X & X\end{array}\right)$ where " $X$ " is as described above.

Note that, all the matrices are following the same pattern and hence having a same shape. [5]:

If we calculate the Determinant of the above matrices

$$
\Delta(X)=\Delta(L) \cdot \Delta(L)=a^{2} \times a^{2}=a^{4}
$$

Likewise, for $Y$, $\Delta(Y)=\Delta(X) \cdot \Delta(X)=a^{4} \times a^{4}=a^{8}$ where the matrix $Y$ has order $=2 \times 2 \times 2=8$

So we can generalized the det. value as

$\Delta(L$-Block $)=a^{n}$

where $n$ is the order of the matrix.

Now, if we more generalize our Block Matrices with different $L$ matrices having different elements, then we can write $X^{\prime}$ as -

$$
\left(\begin{array}{cc}
L_{1}^{\prime} & 0 \\
L_{2}^{\prime} & L_{3}^{\prime}
\end{array}\right)
$$

where $L_{1}^{\prime}, L_{2}^{\prime}, L_{3}^{\prime}$ are $L$ matrices having different elements.

Note, $\Delta\left(L_{1}^{\prime}\right)=a_{1}^{2} \quad \Delta\left(L_{2}^{\prime}\right)=a_{2}^{2} \quad \Delta\left(L_{3}^{\prime}\right)=a_{3}^{2}$ and that is how, the value of

$$
\Delta\left(X^{\prime}\right)=\Delta\left(L_{1}^{\prime}\right) \cdot \Delta\left(L_{3}^{\prime}\right)=a_{1}^{2} \times a_{3}^{2}=\left(a_{1} \times a_{3}\right)^{2}
$$

Again, going for bigger ordered matrices, we have $Y^{\prime}$ which has blocks of $X^{\prime}, X^{\prime \prime}, X^{\prime \prime \prime}$ and if we go through above method, we can find the

$$
\Delta\left(Y^{\prime}\right)=\Delta\left(X^{\prime}\right) \cdot \Delta\left(X^{\prime \prime \prime}\right)=\left(a_{1} \times a_{3}\right)^{2} \times\left(b_{1} \times b_{3}\right)^{2}
$$

Where $a_{1}, a_{2}, a_{3}, \cdots$ are elements of $X^{\prime}$ matrix. likewise, $b_{1}, b_{2}, b_{3}, \cdots$ are elements of $X^{\prime \prime \prime}$. Here we can write $X^{\prime \prime \prime}$ as

$$
\left(\begin{array}{cc}
L_{1}^{\prime \prime \prime} & 0 \\
L_{2}^{\prime \prime \prime} & L_{3}^{\prime \prime \prime}
\end{array}\right)
$$

So, In general, Determinant value of Block "L" matrices can be written as:

$\Delta(\operatorname{Block} L)=\left(a_{1} \cdot a_{3}\right)^{2} \cdot\left(b_{1} \cdot b_{3}\right)^{2} \cdot\left(c_{1} \cdot c_{3}\right)^{2} \cdots$

Where $a, b, c, d, \cdots$ are elements of different "L" Matrices.

\section{Hybrid "L" Matrix}

We can make a matrix in which it has blocks of different kinds of "L" matrices like $L\lrcorner,\rceil, \Gamma$.

They may or may not repeat in the matrix. We are calling this type of matrix as hybrid " $L$ " matrix where the building block of matrix is different types of $\mathrm{L}$ matrix. This type of shapes can be found in the nature itself.

$$
\left(\begin{array}{llll}
a & 0 & 0 & b \\
a & a & b & b \\
c & c & d & d \\
c & 0 & 0 & d
\end{array}\right)
$$

Matrix having all four type of $\mathrm{L}$ matrices.

Here we can see the different $L$ patterns. The elements are arranged in this fashion that they are constructing different L shapes. In future, the Determinant value and inverse of the above matrix can be evaluated.

\section{Conclusion and Future Work}

In this technical report, we reflect on the approach of arriving at structured matrices. Specifically, we propose some concrete approaches to define innovative structured matrices. Furthermore, we define the family of L-matrices and study some of their properties. We expect this class of matrices to find many applications in future.

\section{REFERENCES}

[1] G. Rama Murthy, "Novel Structured Matrices," Interna- 
tional Journal of Algorithms, Computing and Mathematics, Vol. 2, No. 4, 2009, pp. 19-32.

[2] J. Gilbert and L. Gilbert, "Linear Algebra and Matrix Theory," Academic Press, Waltham, 1995.

[3] R. M. Gray, "Toeplitz and Circulant Matrices: A Review," Technical Report, Stanford University, Stanford,
2006.

[4] A. S. Householder, "The Theory of Matrices in Numerical Analysis," Dover Publications, New York, 1975.

[5] T. kailath, "Linear Systems," Prentice-Hall, Inc., Englewood Cliffs, 1980. 